\title{
Valores y formación profesional: miradas al contexto universitario
}

Ethics and professional education, a glimpse at the university context

Valores e formação profissional, um olhar sobre o contexto universitário

\author{
Edelmis Cristina Reyes-Quiñones \\ Centro de Referencia de Educación de Avanzada (CREA) \\ Universidad Tecnológica de La Habana Cujae, Cuba \\ Andrés Avelino Martínez-Morales \\ Universidad de La Habana, Cuba
}

Fecha de recibido: 20/10/2020 • Fecha de aceptado: 13/2/2021

\begin{abstract}
Los valores están presentes en los procesos cotidianos. No solo influyen en cómo se actúa, sino también en la forma con la cual se observa el mundo, determinando las percepciones sobre el comportamiento individual y social de los humanos, como aspiración y conquista, siempre inacabadas, del bien. Todas nuestras acciones están marcadas o reflejan valores establecidos que, a su vez, están condicionados por nuestra forma de reflexionar, nuestras estructuras de pensamientos, nuestras creencias, los aspectos que nos enseñaron y las experiencias que vamos adquiriendo. Este ensayo trata de esta impronta, específicamente, en el ámbito universitario
\end{abstract}

Palabras claves: educación en valores, formación universitaria, Cuba, pensamiento praxeológico y pedagógico.

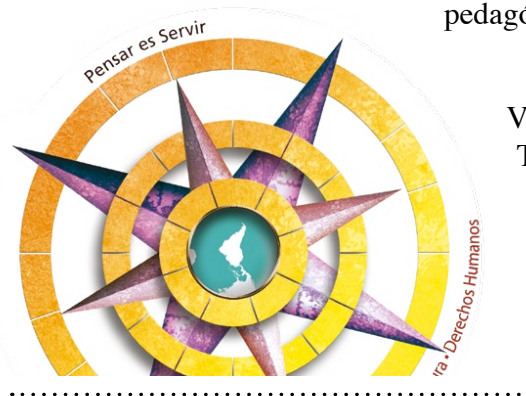

Abstract

Values are present in everyday processes. They not only influence how we act, but also how we observe the world, determining our perceptions of individual and social human 
behavior, as an aspiration and conquest, always unfinished, of the good. All our actions are marked, or reflect, certain values that, in turn, are conditioned by our way of thinking, our thought structures, our beliefs, the aspects we have been taught and the experiences we have acquired. This essay deals with this imprint, specifically in the university environment.

Keywords: ethics in education, university education, Cuba, praxeological and pedagogical thinking.

Resumo

Os valores estão presentes nos processos cotidianos. Eles não só influenciam a forma como agimos, mas também a forma como observamos o mundo, determinando as percepções do comportamento individual e social do ser humano, como aspiração e conquista, sempre inacabada, do bem. Todas nossas ações são marcadas, ou refletem, certos valores que, por sua vez, são condicionados por nossa maneira de pensar, por nossas estruturas de pensamento, por nossas crenças, pelos aspectos que nos foram ensinados e pelas experiências que adquirimos. Este ensaio trata desta impressão especificamente no ambiente universitário.

Palavras chave: valores educação, educação universitária, Cuba, pensamento praxiológico e pedagógico.

\section{Introducción}

\section{[...] Nosotros defendemos la política de paz por una cuestión}

de principio, porque la paz es una aspiración justa de toda la humanidad $[\ldots]^{1}$

(Fidel Castro Ruz, 1967, p. 155)

\section{El tema de los valores está presente} desde los inicios de la humanidad ya que siempre han existido cosas

1 Discurso pronunciado en la concentración conmemorativa del quinto aniversario de la Revolución, en la Plaza de la Revolución, el 2 de enero de 1964. Aniversarios del triunfo de la Revolución Cubana. Editora Política. La Habana. 1967. Pp. 155. preciadas como el bien, la verdad, la belleza, la felicidad, la virtud. Sin embargo, el criterio para darles valía ha variado a través de los tiempos, de acuerdo con la estética, esquemas sociales, costumbres, principios éticos, el costo, la utilidad, el bienestar, el placer, el prestigio.

José Martí Pérez, a lo largo de su obra, trató el tema de los valores desde diferentes aristas, en todos los estilos literarios que trabajó, y señalaba "hay que ir a lo mejor de la gente porque si no, lo peor prevalece". Los valores se traducen en pensamientos, conceptos o ideas,

2 Valores y formación profesional: miradas al contexto universitario Edelmis Cristina Reyes Quiñones, Andrés Avelino Martínez Morales

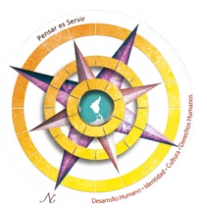


pero lo que más apreciamos es el comportamiento, lo que hace la gente. Una persona vive de acuerdo con los valores en los que cree. Ella vale lo que valen sus valores y la manera con la cual los vive.

El tópico de los valores es tratado hoy de modo reiterativo por muchos en diversos textos, publicados en las diferentes formas de comunicación existentes. No obstante, no todos conocen y dominan los conceptos o significados que se enmarcan en este asunto tan complejo y de necesario conocimiento para lograr una vida social sana y solidaria.

Muchas personas hablan de valores y no tienen claro qué son los valores y cuál es su real significación en la vida cotidiana. Sin embargo, declaran intuitivamente que, en la actualidad, en relación con sus tiempos, hay una crisis de valores (Díaz-Llorca, 2010).

Esta reflexión nos orienta, fundamentalmente, a padres, maestros, profesores, investigadores, comunicadores e historiadores, sobre el rol que corresponde desempeñar en la formación, de las juventudes con las cuales interactúan, al portar los más altos valores que socialmente se reconocen y se quieren alcanzar; su discurso tiene que ser consecuente con su acción, no pueden crear vacíos entre lo que dicen y lo que hacen. Pero, ellos no son los únicos, el Estado e instituciones, a través de programas, deben, con un grupo de psicólogos, sociólogos, pedagogos, buscar alternativas, aprovechando el uso adecuado de las tecnologías.

Es muy importante saber encauzar la vida de los que nos suceden, a través de valores acordes con los principios sociales que garantizan una existencia estable y placentera. Los valores de los niños pequeños están definidos, en buena medida, por sus necesidades de subsistencia y la búsqueda de aprobación de sus padres como sustento biológico y amor filial.

\section{Los valores en la formación}

Existe un grupo de valores que se derivan de la cultura de la sociedad y que es necesario transmitir a través de las generaciones que la conforman. Cada país o región del mundo asume sus valores, en correspondencia con sus creencias y normas, esas que le permiten una forma de vida comunitaria, con estabilidad en el tiempo y coherente con su naturaleza. 
Sin embargo, es necesario conducir la formación de los ciudadanos, desde las primeras edades de vida hasta el momento en que deben asumir la dirección de la sociedad. En esto, juega un papel importante la educación universitaria.

La enseñanza de los valores universales del hombre, como la vida, la libertad, la justicia, la paz y la felicidad, es necesaria desde los inicios. Otros valores es preciso enunciarlos y moldearlos, mediante las distintas etapas que conforman el proceso de enseñanza-aprendizaje. Estos últimos dan indicio de una conducta aceptable de la niñez a la juventud: amabilidad, amistad, bondad, perseverancia, bien común, solidaridad, gratitud, coherencia, responsabilidad, fidelidad, puntualidad, profesionalidad, entre otros.

La amabilidad no nace con el niño, este es impulsivo por naturaleza y a ser amable y cortés se aprende en las más diversas actividades de la vida cotidiana. Los infantes asimilan las normas de comportamiento social en la medida que los adultos los entrenan y enseñan a comportarse de acuerdo con esas normas. La amabilidad implica, a su vez, la igualdad en el tratamiento a los pequeños y la delicada cortesía de unos a otros, por lo que han de aprender a ser amables con todos los compañeros, así como a ayudarles en todo momento.

El juego de roles, en el que es posible modelar las más diversas situaciones en las cuales esté implícito el ser amable y cortés, es una de las vías más importantes para que los niños interioricen estos valores. Es muy efectivo el ejemplo del adulto para enseñar las normas de la amabilidad; por eso, los padres deben ser modelos por imitar en dicho comportamiento.

Como seres sociales, somos todos iguales, aunque nos diferencian algunos valores, según la sociedad donde vivimos. La discriminación puede ocurrir en cualquier ámbito. Por ello, es sumamente trascendental que los infantes sepan cómo no discriminar ni a ver el racismo u otras manifestaciones xenófobas y segregacionistas como algo normal. Desde edad temprana, es preciso comprender que la diversidad existe y que como tal se debe respetar. Conviene el aprendizaje de hacer amigos y respetar a los demás, independientemente de su color de piel, sus rasgos, de cómo es su pelo, si habla otro idioma, su cultura y sus tradiciones. Por esta razón, el respeto a las diferencias, a lo diverso, a las distintas culturas y razas también deben ser parte de la educación que damos a nuestros hijos. Los adolescentes guían sus

4 Valores y formación profesional: miradas al contexto universitario Edelmis Cristina Reyes Quiñones, Andrés Avelino Martínez Morales

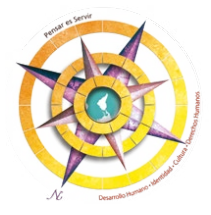


valores personales por su necesidad de experimentación y autonomía, de amistad y libertad.

La amistad es uno de los valores más importantes por desarrollar en la educación. Se trata del afecto personal, puro y desinteresado, compartido con otra persona; nace y se fortalece por medio de las interrelaciones entre los seres humanos. Con el objetivo de que el niño o el joven asimile este valor, es necesario formarle con nociones, conocimientos, habilidades, emociones, vivencias y sentimientos sobre las relaciones interpersonales y que le preparemos para vivir con armonía y respeto. Es fundamental aprender que un buen amigo puede ser para siempre y que, para eso, se requiere cultivar y alimentar la amistad, día tras día, en la escuela, en el parque, en la vecindad, en la universidad, etc. La amistad es un sentimiento sublime, "es el crisol de la vida" (Martí Pérez 1875, p. 2010).

"La bondad es la flor de la fuerza" (Martí Pérez, 1885, p. 198), es una de las cualidades humanas que mejor reflejan su esencia, pues la persona bondadosa es buena, benigna y benévola. A veces se le relaciona con la amabilidad. Ser bondadoso es tener inclinación por hacer el bien a los demás y se lleva a cabo con afecto, comprensión y respeto. La buena persona suele ser bondadosa. Por eso la necesidad de iniciar, cuanto antes, a los niños en conductas bondadosas. El joven que aprende a construir la paz y vivirla en bondad recibirá muchas dádivas a cambio. El modelo que le ofrece el adulto le dirá mucho más que mil palabras; su efecto será más inmediato y profundo si este adulto realiza acciones que revelen comportamientos bondadosos; aprenderá que lo mejor es ayudar, comprender y cooperar, en lugar de agredir, arrebatar o maltratar.

La perseverancia es un esfuerzo continuo, supone alcanzar lo que se propone y buscar soluciones a las dificultades que puedan surgir, un valor fundamental en la vida para obtener un resultado concreto. El joven logra, con perseverancia, la fortaleza y esto le permite crecer y no dejarse llevar por lo fácil y cómodo. El estudio siempre implica paciencia y perseverancia para su conclusión. Por esto, es muy importante que desde edad temprana se aprenda a ser perseverante, porque se irá forjando estabilidad, confianza en sí mismo y madurez.

Martí expresó “... hacer bien es un deber sencillo, que la beneficencia ostenta y alardea, pero que la caridad cumple en silencio" (1875, p. 346). Más adelante, alerta que "es preferible el bien de muchos a la opulencia 
de pocos" (p. 346)., por tanto, el bien común, desde el inicio de la vida y hasta la adolescencia será apropiado en la educación por el respeto a las cosas, en la casa y en la escuela, inclusive en lo que corresponde a los bienes sociales, como los parques, las bibliotecas, los teatros, los monumentos, los museos, los cines, etc., ya que son, igualmente, bienes comunes.

Los padres, abuelos, educadores, maestros deben enseñar a la niñez a no romper ni arrancar plantas del jardín, no tirar papeles en la calle, no romper los juguetes del parque, no estropear el césped ni pintar las paredes. Desde pequeños, debemos cuidar los libros de la biblioteca, no tocar los cuadros ni esculturas $\mathrm{u}$ otros objetos de un museo y no molestar a los animales en el zoológico.

Asimilar el cuidado del medio ambiente e instruir a las personas a reciclar los distintos materiales, para no malgastar los recursos naturales del planeta, es necesario e importante, pero la convivencia en la casa, la escuela, la universidad también refuerza, a través de la educación sistemática, el cuidado de las calles, de las ciudades y del mobiliario urbano, las plazas, los ríos y las fuentes, que son otros bienes comunes y, como tales, deben ser respetados. No es correcto que se tire basura ni restos al suelo, bañarse en las fuentes, hacer ruido en los museos, gritar o escuchar música alta en el ómnibus o lugares públicos, romper los asientos de los teatros o cines.

La solidaridad debe ser enseñada y transmitida, es el ejercicio que más fomenta la interiorización de los valores. Es necesario ponerse en el lugar del otro y eso puede empezar al compartir, asistir, colaborar, ayudar, etc. Ser solidario precisa ser una regla como las demás normas de educación. Es recomendable hablar de lo que está bien y mal, como también de lo que a los otros les gustaría y de qué podrían hacer ellos para colaborar, ayudar o cooperar. La solidaridad es altruismo, es compartir lo que se tiene, se aprende y se afianza tanto con la práctica como con el ejercicio.

La gratitud es uno de los valores más apreciados y respetados a la hora de educar. Se enseña a dar las gracias con el ejemplo. El acto de agradecer es gratuito y no debemos esperar nada a cambio; nunca olvidar dar las gracias por tenderme la mano, dejarme pasar, llamarme, haberme preparado el desayuno, recordar mi cumpleaños. También debemos ser agradecidos con detalles, gestos, sonrisas, besos y abrazos.

6 Valores y formación profesional: miradas al contexto universitario

Edelmis Cristina Reyes Quiñones, Andrés Avelino Martínez Morales

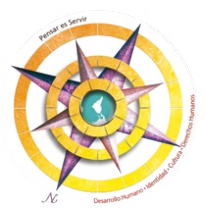


La coherencia es un valor que se transmite con el ejemplo. Los padres deben ser coherentes con lo que dicen y hacen. Si existen contradicciones entre sus palabras y sus actos, estarán creando confusión y dificultades en el seno de su familia. En la educación, el valor de la coherencia se entiende como una característica de la autenticidad en la vida. Es lo que da sentido a la confianza, fidelidad, honestidad, sinceridad y a otros tantos valores que van relacionados. La división, la falsedad, la ruptura entre lo que se piensa, se dice y se hace muestran que falta unidad en la forma de asumir la existencia. Todos debemos realizar un esfuerzo diario por conseguir esa unión, lo que a su vez nos lleva a ser cada vez más coherentes. La unidad en la vida de una persona comprende tanto sus pensamientos como sus obras y no entiende de fisuras entre ambas.

La responsabilidad, como valor social, está ligada a garantizar el cumplimiento de los compromisos adquiridos y a generar tanto confianza como tranquilidad entre las personas. Toda responsabilidad está estrechamente enlazada a la obligación y al deber (ambos conceptos constituyen la materia prima de la responsabilidad). Por tanto, el joven universitario es responsable de sus elecciones y sus actos; de su felicidad personal; de elegir los valores según los cuales vive y de elevar el grado de su autoestima; del modo en que distribuye su tiempo y realiza sus deberes, al igual que sus trabajos pendientes; de hacer realidad sus deseos estudiando cada día más.

La fidelidad, tan ligada al valor de la lealtad, es algo que no debe faltar. Implica compromiso y responsabilidad. Por ello, un joven es fiel cuando no engaña; no miente; asume las consecuencias positivas o negativas de decir la verdad; sabe valorar los vínculos con sus padres, hermanos, abuelos, amigos y compañeros. Ser fiel a padres y abuelos es no intentar engañarles; ser fiel a hermanos y amigos es apoyarles en todo momento, pero, más importante aún, es demostrar fidelidad y ser fiel a uno mismo.

La puntualidad, significa un compromiso para realizar las tareas a tiempo: llegar puntual al aula, a un examen, a una reunión, a una cita con amigos.

\section{Los valores para la vida}

La formación de valores no es un proceso espontáneo, por el contrario, es dirigido. Algunos valores permanecen a lo largo de la vida de las personas, aunque en la edad adulta se originan modificaciones, al 
plantearse nuevas prioridades como la salud, el éxito profesional, la responsabilidad laboral y familiar, la consagración al trabajo.

Podemos considerar que la profesión es la ocupación fundamental del sujeto, con la cual, a partir de los conocimientos, las habilidades y los valores, se resuelven problemas vitales cuya solución se dirige a satisfacer las necesidades de la sociedad.

Profesional, según el Diccionario de la Real Academia Española, indica que es perteneciente a la profesión, la que se ejerce con relevante capacidad y aplicación (Real Academia Española, 2014). La Educación Avanzada identifica como profesional al "[...] individuo que a partir de una instrucción formalizada o no, ejerce una profesión demostrando en su actividad cuatro cualidades que lo identifican, ellas son: rapidez, exactitud, precisión y cuidado" (Añorga, 1997, p. 7).

En un profesional se reconocen aspectos como:

- Dominio teórico-metodológico del objeto de la profesión que le permita la transformación del contexto de actuación y la autotransformación.
- Una ética de la profesión que se manifieste en el desempeño (amor, dignidad, honor, abnegación y entrega).

- Satisfacción personal y profesional por la labor que se realiza (espíritu innovador, transformador, creativo y optimista).

- Una identificación con la profesión que inste a implicarse con responsabilidad en la tarea realizada, asumiendo los riesgos y éxitos que implica su ejercicio, de forma honesta, modesta y sencilla (autenticidad en el actuar, plenitud, libertad, a partir de la sinceridad).

- Formar parte de asociaciones profesionales (lucha incansable por el perfeccionamiento constante y la excelencia de su trabajo).

A partir de estos elementos, asumimos que ser profesional es contenido fundamental de la profesionalidad como valor. Es el cúmulo de conocimientos, experiencias, sentido común, actitudes, aptitudes, habilidades, creatividad, innovación, empuje, dinamismo y otras tantas características.

Los profesionales (Núñez, 2007) que no posean una buena formación,

8 Valores y formación profesional: miradas al contexto universitario

Edelmis Cristina Reyes Quiñones, Andrés Avelino Martínez Morales

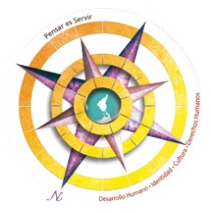


susceptible de permanente actualización, quedarán marginados en lo económico, social y cultural. El saber científico vinculado a los procesos productivos o de servicios genera resonancias económicas, éticas y políticas (Núñez, 2000), por lo que el conocimiento del profesional se convierte en un insumo principal (Lage, 2004) de los procesos productivos o de servicios en los que participa.

Este valor con un carácter más integral, dialéctico, de interacción de factores internos y externos, donde su función capital es educar, formar, incluyendo la instrucción. Todo esto es importante considerarlo no solo en la profesión pedagógica.

La profesionalidad pedagógica contiene en su concepto elementos validos no solamente para la profesión, sino para la profesionalidad en cualquier faceta, como los valores, las cualidades, los conocimientos, los métodos y habilidades profesionales avalados por las experiencias, los resultados y los logros obtenidos. Es una manera de hacer, un modo de actuar, que pone de manifiesto no solo las competencias de quien la desarrolla, sino las mejores virtudes asociadas al ejercicio de la profesión. Acumula valores, habilidades, resultados, que se transmiten y adquieren con el estudio y la experiencia, en las diferentes actividades profesionales del ser humano.

La división social y profesional del trabajo determina la existencia de particularidades en la moral de los diferentes oficios y profesiones. Esta moral profesional es el objeto de estudio de la ética profesional. Chacón (2005) la define como una rama de la ética, la cual estudia la forma específica con que se manifiesta la moral de la sociedad en la moral de las profesiones. Fundamenta los mecanismos de la regulación moral; formula el código de principios y normas morales de la profesión, al sistematizar la moralidad de los grupos profesionales; establece el ideal de la imagen social de la personalidad del tipo de profesional al que aspira la sociedad o que esta necesita.

El propio desarrollo de la ciencia, la técnica y la tecnología, unido al proceso de surgimiento y expansión de las universidades (cuya misión es formar profesionales), aparejado al desenvolvimiento actual de la economía de mercado (en las condiciones de la globalización neoliberal), hace extensiva la necesidad de implementar la moral de las profesiones y de la ética profesional; así, han aparecido códigos de ética de profesiones específicas en los diferentes países 
del planeta. La ética profesional es la ciencia normativa que estudia los comportamientos morales debidos y exigibles en toda profesión; involucra el código de normas que ayuda al profesional a saber actuar correctamente en todo momento; contiene los principios y valores que se estiman requeridos para ejercer una profesión.

El concepto ético humanista de la profesionalidad destaca un modo de actuación caracterizado por un saber ser para saber hacer en la vida familiar, laboral y social, desde los aprendizajes básicos. Por tanto, el hombre es el valor supremo de la sociedad, como sujeto activo, crítico y transformador consciente de su realidad y de sí mismo, en condiciones de dignidad, justicia, cultura, creatividad e independencia.

La profesión y la profesionalidad ${ }^{2}$ guardan estrecha relación con la continuidad y la intensidad de la formación de los trabajadores, en el logro de conocimientos, habilidades técnicas y valores compartidos integralmente.

En su intervención en el Congreso Internacional de Pedagogía 2011,

2 La profesionalización del maestro, según Fernández A. M, 1996, implica utilizar la ciencia pedagógica como recurso fundamental para interpretar, comprender y dirigir el proceso de enseñanza-aprendizaje. el ministro de educación superior, Miguel Díaz-Canel, reafirmaba que "nuestros procesos formativos integran los conceptos de calidad, racionalidad, eficiencia y pertenencia para lograr la formación integral de los profesionales" (Díaz-Canel, 2011, p. 8).

De acuerdo con diferentes autores consultados, enseñanza es la actividad que garantiza la apropiación activa y creadora de la cultura, en la que se intercambian, recrean y crean significados, sentimientos y modos de actuación, los cuales permiten comprender la realidad objetiva y subjetiva, así como actuar sobre ella para adaptarse o transformarla. Asimismo, la enseñanza facilita y potencia los aprendizajes desarrolladores o formativos; propicia experiencias afectivas, estimulando la formación de sentimientos, actitudes, normas y valores, de forma planificada y organizada.

A través de la enseñanza, se fortifica no solo el aprendizaje, sino también el desarrollo humano. El comportamiento humano es un $99 \%$ de imitación, por consiguiente, la manera de educar las virtudes y los valores será, fundamentalmente, con el ejemplo, con la vivencia personal de cada uno de ellos, porque, según el adagio latino, las palabras mueven, pero los

10 Valores y formación profesional: miradas al contexto universitario Edelmis Cristina Reyes Quiñones, Andrés Avelino Martínez Morales

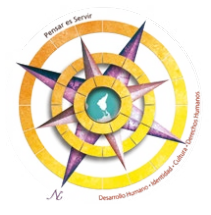


ejemplos arrastran. Es importante que los estudiantes vean que los profesores hacen lo que dicen.

Las definiciones del proceso de enseñanza-aprendizaje consultadas en la literatura científica, pedagógica, conducen a la secuencia sistémica de acciones desarrolladoras, conscientemente coordinadas entre el profesional de la educación, el estudiante, el grupo y los otros factores que tienen incidencia sobre el desarrollo de la personalidad. La orientación moral, el sentido de continuidad, las explicaciones acerca del pasado y la claridad sobre el presente impulsan la solución de contradicciones que se manifiestan en la formación inicial. De esta última depende el ascenso a niveles más altos de autorregulación, autodeterminación en el modo de actuación profesional, desde el dominio de contenidos científicos y técnicos, contribuyendo a la transformación de los individuos en especialistas comprometidos con su propio desarrollo.

La educación en la profesionalidad de la enseñanza-aprendizaje debe ser fundamentada sobre la base de los referentes más novedosos de las ciencias pedagógicas, (leyes, principios, así como sus componentes estructurales). Enuncia la manifestación de ciertas relaciones dialécticas en el marco de las ciencias, que deben concretarse en la didáctica del proceso. Entre ellas, los vínculos ciencia-profesión, teoría-práctica, ciencia-tecnología-sociedad, así como formas de trabajo interdisciplinar, consecuencia de los procedimientos de integración-diferenciación ocurridos entre las disciplinas científicas. La enseñanza-aprendizaje de la profesión requiere la articulación armónica de los componentes del currículo (laboral, académico e investigativo). Por tanto, la educación en el valor de la profesionalidad en el proceso de enseñanza-aprendizaje devela el aspecto sociohumanista que subyace en las ciencias aplicadas, pedestal para el desarrollo de una cultura de la misma tecnología apropiada.

En términos del valor de la profesionalidad también es importante hacer referencia a la relación ética y el desarrollo de un programa económico social que sea congruente con aquélla.

Se evidencia que la economía política no puede ser ajena a la ética, al desarrollo moral de los individuos.

La educación superior cubana ha logrado conjugar conocimientos, cultura, ciencia, tecnología con sociedad, masividad, comunidad, territorialidad, hombre; de ahí que hoy se sienta orgullo al decir que nuestro sistema 
educacional es propio, novedoso y paradigma por seguir para otros pueblos americanos y del mundo. Tiene inaplazables retos que cumplir; uno de ellos es reforzar el rescate y la formación de valores, a la altura de los objetivos más sagrados que persiguen el socialismo y la patria.

\section{A manera de reflexión final}

Los valores son la base para vivir en comunidad y relacionarnos con las demás personas. Permiten regular nuestra conducta para el bienestar colectivo y una convivencia armoniosa.

En la sociedad los diferentes agentes, por ejemplo la escuela y los amigos, influyen y hasta modifican nuestras percepciones. También son portadores externos de nuestra cultura universal los lugares que se visiten, una biblioteca, un museo, un centro deportivo, al igual que las agencias que transmiten informaciones, noticias y entretenimiento. Los periódicos, las revistas, las televisoras son fuentes importantes, ya que son públicas, están al alcance de todos y traspasan las más diversas corrientes de creencias y valores.

Los valores en el contexto universitario educan en la profesión y la profesionalidad, ya que el ser humano es el bien supremo de la sociedad, como sujeto activo, crítico y transformador, consciente de su realidad y de sí mismo, en condiciones de dignidad, justicia, cultura, creatividad e independencia.

12 Valores y formación profesional: miradas al contexto universitario Edelmis Cristina Reyes Quiñones, Andrés Avelino Martínez Morales 


\section{Referencias bibliográficas}

Addine, F. y García, G. (2003). Profesionalidad y actividad investigativa del docente. Pedagogía Curso 54. Soporte electrónico.

Alarcón, R. (2013). La calidad de la educación superior cubana: retos contemporáneos. La Habana: Editorial Félix Varela.

Álvarez de Zayas, C. (1999) La escuela en la vida. La Habana: Editorial Pueblo y Educación.

Añorga, J. (1997). La profesionalización y la educación avanzada. Cuba: Instituto Superior Pedagógico Enrique José Varona.

Arzuaga, M. (2011) Modelo para la formación de proyectos de vida profesionales en los estudiantes de las especialidades técnicas de la licenciatura en educación. [Tesis presentada en opción al grado científico de Doctor en Ciencias Pedagógicas. La Habana. Cuba].

Batista, N. (2001) Una concepción metodológica de educación en valores para su diseño curricular en las carreras de ingeniería. [Tesis en opción al grado científico de Doctor en Ciencias Pedagógicas. Ciudad de $\mathrm{La}$ Habana].

Báxter, E.(2002). La escuela y la formación del hombre. En Compendio de Pedagogía. La Habana: Editorial Pueblo y Educación. pp. 143-192.

(2002) Educación en valores. En Compendio de Pedagogía. La
Habana: Editorial Pueblo y Educación. pp. 193-199.

Bernaza, G; Troitiño, D. y López, Z. (2018). La superación profesional: mover ideas y avanzar más. La Habana, Editorial Universitaria. MES.

Blanco Pérez, A. (2002). La educación como función de la sociedad. En González Soca, A M. (Ed.). Nociones de Sociología, Psicología y Pedagogía. La Habana: Editorial Pueblo y Educación. pp. 4-21.

Bombino, L. (2004). ¿De qué Ética hablamos?. La Habana: Editorial Félix Varela.

Borrego, L. (2005) Una estrategia para educar el valor laboriosidad en los alumnos de Secundaria Básica. [Tesis presentada en opción al grado científico de Doctor en Ciencias Pedagógicas. Pinar del Río].

Cánovas, L. y Chávez, J. (2008) Problemas contemporáneos de la Pedagogía en América Latina. Compendio de Pedagogía. La Habana: Editorial Pueblo y Educación.

Cárdenas, N. (2005) Modelo pedagógico para el auto perfeccionamiento del modo de actuación profesional relacionado con la superación que realiza el maestro primario desde el ejercicio de su profesión. [Tesis doctoral. ISP. Félix Varela. Villa Clara. Cuba].

Castro, F. (2011) Concepto de Revolución. Lineamientos de la Política Económica y Social del Partido y la 
Revolución. VI Congreso del PCC. La Habana. 2011.

(2004) Las ideas creadas y probadas por nuestro pueblo no podrán ser destruidas. Discurso en la clausura del Cuarto Congreso de la Educación Superior. Cuba: Oficina de Publicaciones del Consejo de Estado. pp.18-22.

(2003) Discurso de clausura del Quinto Encuentro Internacional de Economistas sobre Globalización y Problemas del Desarrollo. La Habana: Oficina de Publicaciones del Consejo de Estado.

(1999) Una revolución solo puede ser hija de la cultura y las ideas. Discurso pronunciado el 3 de febrero, en el Aula Magna de la Universidad Central de Venezuela. La Habana: Editora Política.

(1999) Capitalismo actual, características y contradicciones: neoliberalismo y globalización. Selección temática 1991-1998. La Habana: Editora Política.

Castellanos, D. (2001) Educación, aprendizaje y desarrollo. Curso 16. La Habana; Congreso de Pedagogía.

Chacón, N. (2005) Moralidad histórica y valores en la sociedad. Temas actuales de la política, la ideología, la ciencia, la tecnología y la cultura. [Maestría en Ciencias de la Educación]. Cuba:IPLAC..Soporte electrónico.

(2000) Humanismo y valores en la formación del profesional de la educación: el componente humanista y la formación de maestros cubanos. Ciencia Y Sociedad, 25(4), 490-533. https://doi.org/10.22206/ cys.2000.v25i4.pp490-533

(2002) Dimensión Ética de la educación cubana. La Habana: Editorial Pueblo y Educación.

(1999) Ideario pedagógico de José de la Luz y Caballero. La Habana; Editorial Pueblo y Educación.

(1996) Formación de valores morales. La Habana: Editorial Academia.

Chávez, J. (2002). Bosquejo histórico de las ideas educativas en Cuba. La Habana: Editorial Pueblo y Educación.

Colectivo de autores. (1999). Formación de Valores en las Nuevas Generaciones. La Habana: Editorial Ciencias Sociales.

Díaz Canel-Bermúdez, M. (2008). Declaración de la Conferencia Regional de Educación Superior. La Habana: Centro de Estudios de la UCP "Enrique José Varona".

Díaz, C. (2010) Cómo utilizar una estrategia de valores en las organizaciones. El lado oculto de los equipos gerenciales. Caracas: OPSU, Fondo Editorial.

Real Academia Española (2017). Diccionario de la Lengua Española. Tomos I y II. Madrid: Editorial Espasa Calpe S.A.

Fabelo J.(1996) La formación de valores en las nuevas generaciones. La Habana: Editorial Ciencias Sociales.

14 Valores y formación profesional: miradas al contexto universitario Edelmis Cristina Reyes Quiñones, Andrés Avelino Martínez Morales

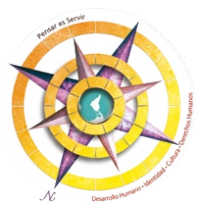


(2003) Los valores y sus desafios actuales. La Habana: Editorial José Martí.

(2011) Discurso de apertura en el Evento Universidad. La Habana: CEPES.

García, G.(2008) Compendio de Pedagogía. La Habana: Editorial Pueblo y Educación.

González, V. (2004). El profesorado universitario: su concepción y formación como modelo de actuación ética y profesional. Revista Iberoamericana De Educación, 34(1), 1-11. https:// doi.org/10.35362/rie3412934

(1999) El profesor universitario, ¿un facilitador o un orientador en la educación de valores? En Revista Cubana de Educación Superior.

(1994) Motivación profesional y personalidad. Colombia; Editora Universitaria Sucre.

González, M. (2001) Los valores morales en el técnico cubano contemporáneo. [Tesis en opción al grado científico de Doctora en Ciencias Filosóficas. Ciudad de La Habana].

Guadarrama, P (1985). El pensamiento filosófico de José A. Caballero, Félix Varela, José de la Luz y Caballero. En Valoraciones sobre el pensamiento filosófico y latinoamericano. La Habana, Editorial Política.

Guevara, E. (1965) El socialismo y el hombre en Cuba. La Habana; Editorial Revolucionaria. Hart, A. (200) Una interpretación de la historia de Cuba desde el 2001. Colección
Pensamiento. La Habana: Oficina del Programa Martiano.

Lage, A. (2012). Las funciones de la ciencia en el modelo económico cubano: intuiciones a partir del crecimiento de la industria biotecnológica. Economía y Desarrollo, 147 (1),80-106

Leóntiev, N. (1975) Actividad Conciencia y Personalidad. La Habana: Editorial Pueblo y Educación.

Llanderas, P. y Méndez, M. (2005) Educar en valores: Educación para la salud. Madrid.

Martí, J. (1990) Ideario Pedagógico. La Habana: Editorial Pueblo y Educación.

(1975). Obras Completas. Tomos 7, 8, 11 y 13. La Habana: Editorial de Ciencias Sociales.

(1974) Discurso con todos y para el bien de todos. La Habana: Editorial.

(1875). Galería del senado. La Habana: Centro de estudios Martianos.

Mendoza, L. (2003) Filosofía y axiología en José Martí. Fundamentos de su concepción de la Educación. Revista Varona, 36-37.

(2005) La formación de valores, un proceso complejo. Tema problemas actuales de la educación. CD-ROM. Cuba: IPLAC. 2005 .

Ministerio de Educación (1990) Institutos Superiores Pedagógicos. Programas directores para elaborar el plan de estudio $C$. 
(2003a) La Universalización de los Institutos Superiores Pedagógicos (Prioridades para el curso 2003-2004), Reunión Preparatoria Nacional, mayo. La Habana.

(2003b) Modelo

profesional del Profesor General Integral de la Escuela Secundaria Básica. La Habana. (Documento electrónico).

(2002) Transformaciones en los Institutos Superiores Pedagógicos a partir del curso 20022003, Reunión Preparatoria Nacional, mayo. La Habana.

(2000). Documentos oficiales. Material digitalizado. Modelo del profesional. VRD. UCP "Enrique José Varona". 2000.

MES (2003). Modelo de acompañamiento-apoyo, monitoreo y evaluación del Proyecto Regional de Educación para América Latina y el Caribe. La Habana: PRELAC.

Miranda, F. (1984). Félix Varela: Su pensamiento político y su época. La Habana: Editorial de Ciencias Sociales

Nieto, L. (2005). Modelo de superación profesional para el perfeccionamiento de las competencias profesionales en la actividad educativa con profesores de los Institutos Superiores Pedagógicos. [Tesis en opción al grado científico de Doctor en Ciencias Pedagógicas. Instituto Superior Pedagógico Félix Varela, Santa Clara, Cuba].

Núñez, J. (2007). Democratización de la ciencia y geopolítica del poder.
Quién decide quién se beneficia. Cuba: Editorial Félix Varela.

Núñez, J. (2000). La ciencia y la tecnología como procesos sociales. Lo que la educación científica no debería olvidar. Cuba: Editorial Félix Varela.

Popa, I. y Torres, H. (2006). La educación en valores: premisa indispensable para el futuro profesional cubano de esta época. VII Seminario Nacional para Educadores.

Robbins, S. (2000). Comportamiento organizacional, teoría y práctica. California: San Diego State University.

Sánchez, A., Sánchez, M. (2002). La pedagogía cubana: sus raíces y logros. En Compendio de Pedagogía. pp. 36. La Habana: Editorial Pueblo y Educación.

UNESCO-ORLEAC (2003) Modelo de Acompañamiento-apoyo, monitoreo $y$ evaluación del Proyecto Regional de Educación para América Latina y el Caribe. Aporte al Congreso "Pedagogía 2003”. La Habana: PRELAC

Valle, A. (2002) Algunas consideraciones sobre la transformación de la escuela actual. En Compendio de Pedagogía. La Habana: Editorial Pueblo y Educación.

Vigotsky, L. (1989). Obras Completas. Tomo 5. La Habana: Editorial Pueblo y Educación.

16 Valores y formación profesional: miradas al contexto universitario Edelmis Cristina Reyes Quiñones, Andrés Avelino Martínez Morales

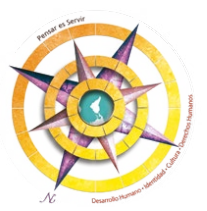

